\title{
Uncertainty Estimation of Refrigeration Equipment Using the Information Approach
}

\author{
Boris Menin \\ Refrigeration Consultancy Ltd., Beer-Sheba, Israel \\ Email:meninbm@gmail.com
}

How to cite this paper: Menin, B. (2020) Uncertainty Estimation of Refrigeration Equipment Using the Information Approach. Journal of Applied Mathematics and Physics, 8, 23-37.

https://doi.org/10.4236/jamp.2020.81003

Received: October 30, 2019

Accepted: December 27, 2019

Published: December 30, 2019

Copyright $\odot 2020$ by author(s) and Scientific Research Publishing Inc. This work is licensed under the Creative Commons Attribution International License (CC BY 4.0).

http://creativecommons.org/licenses/by/4.0/

(c) (i) Open Access

\begin{abstract}
Aims: The purpose of this work is to present the information approach as the only effective tool that allows us to calculate the uncertainty of any result of the study on the use of refrigeration equipment. Methodology: Using the definitions and formulas of information theory and similarity theory, the amount of information contained in a model of refrigeration equipment or process is calculated. This allows us to present formulas for calculating the relative and comparative uncertainties of the model without additional assumptions. Based on these formulas, the value of the inevitable threshold of the accuracy of the representation of the studied construction or process is determined. Results: Theoretically substantiated recommendations are formulated for choosing the most effective methods for analyzing refrigeration equipment are formulated. Conclusion: Having calculated the amount of information contained in the model, we presented practical methods for analyzing data on refrigeration equipment.
\end{abstract}

\section{Keywords}

Information Theory, Refrigeration Equipment, Technical Feasibility, Uncertainty, Ice Slurry, Energy Storage

\section{Introduction}

The refrigeration industry plays an important and growing role in the modern global economy, making a significant contribution in the areas of food, health, energy and environmental protection that policy makers should take better account of.

The International Institute of Refrigeration estimates that the total number of cooling, air-conditioning and heat pump systems operating in the world is about 3 billion. Annual global sales of such equipment amount to approximately 300 
billion US dollars. Nearly 12 million people work worldwide in the refrigeration sector, which consumes about $17 \%$ of the total electricity used in the world.

To use a refrigeration unit with high energy efficiency, it is customary to carry out a careful technical feasibility and research study (TFRS) which carried out at a given time interval and with a predetermined budget. In this case, the developer has a dilemma: take into account a small or large number of quantities. However, what does "a lot" mean and what does "a little" mean? As the Russian poet Mayakovsky noted: two hairs on a bald spot are few, and two hairs in a cup of coffee are many. At present, most scientists believe that with a significant number of quantities, more accurate results can be obtained, forgetting an important fact: any quantity corresponds to some uncertainty, which should be taken into account in the overall uncertainty of the model of the phenomenon under study. However, with an increase in the dimension of the model, only the reliability of the model results improves [1]. Why is this question so important? Because research should be effective both in time and in cost.

In fact, at the moment in all these TFRSs, there is not enough information about their accuracy, or, in other words, with what value of uncertainty of the final result recommendations are made. Any mistake can lead to losses of tens, hundreds and even millions of dollars.

To verify the real situation, scientific and technical articles, reports and studies of the refrigeration equipment and technological processes (TFRS), published in the International Journal of Refrigeration for the period of 2014 to 2019 (1569 units), were analyzed (using SCI-HUB tool) by three criteria simultaneously: a. presentation of results comparing the experimental data with theoretical and/or computer modeling; $b$. the presence of calculations of measurement absolute or relative uncertainties; c. the announcement of the intervals of changes of the main studied quantity and other quantities taken into account in the model. According to these criteria, only $317(20.2 \%)$ publications were selected. The list of papers can be provided.

Most authors note that the model was verified by comparing the theory and experimental results, and the mismatch between them is not significant. However, it looks that most scientists are immersed in physical principles, complicated mathematical formulas and extensive experimental data of the day and take these assumptions as reality, not realizing with what actual accuracy they present their results.

Sometimes, authors declare "uncertainty analysis" or "instrumental uncertainties" chapter. Factually, they only introduce with what accuracy they measure different recorded quantities [2] [3] [4] [5] [6], or the author estimates uncertainty as a result of dividing numerical data by experimental results [7]. Only in one article [8] was a detailed calculation of the total uncertainty of the main investigated quantity given.

An analysis of most publications gives the impression that the authors are undoubtedly confident in the veracity of the presented results. Of course, when 
interpreting data about the studied object-refrigeration equipment or heat and mass transfer process-the developer is expected to hear that the presented model is confirmed by comparing the theory and practice, and the calculation of the achieved measurement uncertainties is given. In fact, in most publications there is no data on the calculated values of the general uncertainty when measuring the observed variable or dimensionless complex. Only one study [9] formulated a general concept for calculating various types of uncertainties and their impact on model accuracy. In the analysed researches, there was no comparison of the experimental uncertainty of the parameter under study (EU) (its definition is introduced in [10]) with the mismatch between the theoretical (TD) and experimental data (ED). If EU > |TD - ED| (for example, it can use mean relative error [11], the average relative deviation [12], the mean absolute percentage error [13] or the mean absolute deviation [14] [15] [16]), the value of the formulated model is doubtful, and putting it into practice is risky. Who benefits from this situation? The developer benefits: she or he increases his own citation index. For this reason, the researchers, having developed seemingly reliable simulation models, for reinsurance, increase the design capacity of, for example, a compressorcondenser unit or the storage tank volume by at least $20-40 \%$. In this case, it is difficult to hope that the results of published studies will find a real practical application.

Thus, a disappointing conclusion suggests itself. Many researchers either do not have sufficient knowledge of the application of measurement theory or simply neglect the use of validation and verification methods [17] and uncertainty analysis [18]. Without a doubt, these methods allow scientists and engineers to identify the relationship between the technical parameters taken into account and the energy efficiency of refrigeration units. The presented situation differs sharply from that when research teams analyze the presence of oil in a certain area or predict heating of the spacecraft hull upon entering the Martian atmosphere [19] [20]. In these cases, the researchers closely examine all possible sources of error, calculate the discrepancy between the theoretical experimental results, and find out the ratio of EU and |TD - ED|.

That is why the objectives of the article are: a) Description of the procedure for applying the information approach; b) Presentation of the calculation of the smallest achievable uncertainty of the model when formulating recommendations to TFRS on choosing the optimal refrigeration unit for a specific consumer; c) Issuing recommendations on the construction of a model for analyzing the operation of refrigeration devices using specific examples.

This method is theoretically justified and has already found numerous applications in quantum mechanics [21], experimental physics [22], and cosmology [23]. Well-defined problems, proposed solutions, and the scope and rationale of the work done are presented to provide an actual background.

\section{Essence of the Information-Oriented Approach}

Any model contains a certain amount of information that allows you to choose 
the optimal number of quantities taken into account. The following is a simplified short introduction to the above statement [24].

Obviously, when formulating TFRS, the designer must develop a model that takes into account certain features of the refrigeration unit or the process under study. In this case, the developer, by default, uses quantities from the existing International System of Units (SI), which includes both base and derived quantities, on the basis of which various classes of phenomena $(C O P)$ are built [25]. For example, $C o P_{\mathrm{SI}}=L M T \Theta$ is used to describe heat and mass transfer processes, where $L$ is length, $M$ is mass, $T$ is time, and $\Theta$ is temperature. There are three additional base quantities: $I$ is the amount of electric current, $J$ is luminous intensity, and $F$ is the amount of substance.

The dimension of the derived quantity $(q)$ is calculated by the following formula [26] [27]

$$
q \ni L^{l} \cdot M^{m} \cdot T^{t} \cdot I^{i} \cdot \Theta^{\Theta} \cdot J^{j} \cdot F^{f}
$$

where $l, m, \cdots, f$ are the base quantities exponents, which vary in the following intervals [28]:

$$
\begin{aligned}
& -3 \leq l \leq+3,-1 \leq m \leq+1,-4 \leq t \leq+4,-2 \leq i \leq+2, \\
& -4 \leq \theta \leq+4,-1 \leq j \leq+1,-1 \leq f \leq+1 .
\end{aligned}
$$

Given (2), you can calculate the values of $e_{l}, e_{m}, \cdots, e_{f}$, which are the number of options of the dimensions for each main quantity

$$
e_{l}=7 ; e_{m}=3 ; e_{t}=9 ; e_{i}=5 ; e_{\theta}=9 ; e_{j}=3 ; e_{f}=3 \text {. }
$$

The total number of dimensionless complexes $\mu_{\mathrm{SI}}$ contained in SI is

$$
\mu_{\mathrm{SI}}=\left(e_{l} \cdot e_{m} \cdot e_{t} \cdot e_{i} \cdot e_{\theta} \cdot e_{j} \cdot e_{f}-1\right) / 2-7=38265
$$

where $\mu_{\mathrm{SI}}$ is calculated in accordance with the $\pi$-theorem [25], and " -1 " is due to the fact that $e_{l}, e_{m}, \cdots, e_{f}$ are equal to zero [24] [25], which corresponds to the case when the quantity has no dimension. Since the SI contains direct and inverse values, for example, length $\left(L^{1}\right)$ and running length $\left(L^{-1}\right)$, the total number of quantities must be divided by 2 . This is because an object having symmetrical parts can be calculated from its one part, while others are considered informationally empty. As already noted, the SI contains seven basic quantities.

The model of the observed/investigated refrigeration unit or technological process contains a certain amount of information $\Delta A_{e}$ calculated by the formula $[24]$

$$
\Delta A_{e}=\kappa \cdot \ln \left[\mu_{\mathrm{SI}} /\left(z^{\prime \prime}-\beta^{\prime \prime}\right)\right]
$$

where $\Delta A_{e}$ is expressed in units of entropy [29], $z^{\prime \prime}$ and $\beta^{\prime \prime}$ are, respectively, the number of all and base quantities registered in TFRS.

To calculate amount of information in bits $\Delta A_{b}$ embedded in a model, it is necessary to divide $\Delta A_{e}$ by $\kappa \ln 2=9.569926 \times 10^{-24} \mathrm{~kg} \cdot \mathrm{m}^{2} \cdot \mathrm{s}^{-2} \cdot \mathrm{K}^{-1} \quad$ [30] [31]:

$$
\Delta A_{b}=\ln \left[\mu_{\mathrm{SI}} /\left(z^{\prime \prime}-\beta^{\prime \prime}\right)\right] / \ln 2(\text { bits })
$$

Based on (6), when comparing different versions of TFRSs used to study the 
same refrigeration unit or technological process, you can identify the most informative version using the following formula:

$$
\begin{aligned}
\Delta A_{b 12} & =\ln \left[\mu_{\mathrm{SI}} /\left(z_{1}^{\prime \prime}-\beta_{1}^{\prime \prime}\right)\right] / \ln 2-\ln \left[\mu_{\mathrm{SI}} /\left(z_{2}^{\prime \prime}-\beta_{2}^{\prime \prime}\right)\right] / \ln 2 \\
& =\left(\ln \left[\left(z_{2}^{\prime \prime}-\beta_{2}^{\prime \prime}\right) /\left(z_{1}^{\prime \prime}-\beta_{1}^{\prime \prime}\right)\right]\right) / \ln 2 \text { (bits) }
\end{aligned}
$$

where $\Delta A_{b 12}$ is an information amount difference between two TFRSs describing the same refrigeration unit by different models, however, belonging to the same $\mathrm{CoP} ; z_{1}^{\prime \prime}$ and $\beta_{1}^{\prime \prime}$ are, respectively, the number of quantities and the number of the base quantities in TFRS ${ }_{1} ; z_{2}^{\prime \prime}$ and $\beta_{2}^{\prime \prime}$ are, respectively, the number of quantities and the number of the base quantities recorded in TFRS ${ }_{2}$. If $\Delta A_{b 12}<0$, then $\mathrm{TFRS}_{2}$ contains more detailed information about the refrigeration system under study. If $\Delta A_{b 12}>0$, then TFRS 1 is preferred. This is due to the fact that in accordance with formula (7), when modeling a physical process, it is preferable to use a model with the number of variables close to optimal, which is due to the choice of a specific class of phenomena depending only on the knowledge, experience and intuition of the researcher.

For example, two research teams analyze the operation of the same refrigeration unit. Results vary. Who presented the most plausible option? Equation (7) is only a preliminary (sufficient) condition for choosing the preferred option. In addition to it, we can formulate the necessary condition [24]: when modeling a physical phenomenon, a system of base quantities is specified, in which there are $\Psi$ dimensional quantities and $\xi$ of base quantities. The developer chooses the target dimensionless basic quantity $u$, the values of which are located in the interval $S$, and specifies the class of phenomena with the total number of dimensional quantities $z^{\prime}$ and the number of base quantities $\beta^{\prime}$. In this case, for a given number of dimensional quantities $z^{\prime \prime}$ and a selected number of base quantities $\beta^{\prime \prime}$ in a model, the absolute uncertainty $\Delta_{\mathrm{pmm}}$ when measuring $u$ is determined by the relation:

$$
\Delta_{\mathrm{pmm}}=S \cdot\left[\left(z^{\prime}-\beta^{\prime}\right) / \mu_{\mathrm{SI}}+\left(z^{\prime \prime}-\beta^{\prime \prime}\right) /\left(z^{\prime}-\beta^{\prime}\right)\right]
$$

Thus, the comparison of different TFRSs can be organized using formulas (7) and (8). At the same time, it should be noted that the total (integral) uncertainty of the model, including uncertainties due to simplification of the original object or phenomenon, as well as uncertainties caused by the transition from the mathematical model to the numerical method, will be significantly larger than $\Delta_{\mathrm{pmm}}$.

It is interesting to note that $\Delta_{\text {pmm }}$ does not depend on the measurement process. It is due only to the decision of the developer to choose a particular class of phenomenon and the number of quantities used to describe the observed process.

In practice and in theory, Equation (8) is a kind of uncertainty relation (correspondence principle) for the model development process. In the study of a physical phenomenon, technological process, or analysis of equipment design, the model must satisfy relation (8). In other words, changing the level of detailed description of the observed object by choosing the class of the phenomenon 
$\left(z^{\prime}-\beta^{\prime}\right)$ and the specific number of variables to be taken into account $\left(z^{\prime \prime}-\beta^{\prime \prime}\right)$ causes a change in the smallest value of the comparative uncertainty $\Delta_{\mathrm{pmm}} / S$ of the main studied function (main variable). Thus, the correspondence principle uniquely determines the achievable accuracy limit (for a given class of phenomena), while simultaneously revealing a pair of quantities observed by a conscious researcher, in particular, absolute uncertainty in the measurement of the studied quantity and the interval of its change.

By implementing Equation (8), we can find the optimal number of dimensionless criteria that is used to calculate the smallest comparative uncertainty of the model corresponding to the selected class of model phenomenon.

We calculate the value of the comparative uncertainty $\varepsilon_{L M T \theta}$ inherent in $\operatorname{Co} P_{\mathrm{SI}}$ $\equiv L M T \Theta$, which is usually used in air conditioning and refrigeration. To do this, one must find the derivative of $\Delta_{\mathrm{pmm}} / S(8)$ with respect to $z^{\prime}-\beta^{\prime}$ and equate the resulting expression to zero:

$$
\left(z^{\prime}-\beta^{\prime}\right)^{2} / \mu_{\mathrm{SI}}=\left(z^{\prime \prime}-\beta^{\prime \prime}\right) \text {. }
$$

By using (3), (4) and (9), $\varepsilon_{L M T \theta}$ will be verified by calculating $z^{\prime}-\beta^{\prime}$ and $z^{\prime \prime}-\beta^{\prime \prime}$ :

$$
\begin{aligned}
& \left(z^{\prime}-\beta^{\prime}\right)=\left(e_{l} \cdot e_{m} \cdot e_{t} \cdot e_{\theta}-1\right) / 2-4=846, \\
& \gamma_{L M T \theta}=\left(z^{\prime \prime}-\beta^{\prime \prime}\right)=\left(z^{\prime}-\beta^{\prime}\right)^{2} / \mu_{\mathrm{SI}} \approx 19,
\end{aligned}
$$

where $\gamma_{L M T \theta}$ is an optimal number of the dimensionless criteria inherent in $C o P_{\mathrm{SI}}$ $\equiv L M T \Theta$, "- 1 " is due to the fact that $e_{b} e_{m}, e_{t}$ and $e_{\theta}$ are equal to zero in expressions (1) and (2). The calculation of the number of dimensionless criteria for $C o P_{\mathrm{SI}} \equiv L M T \Theta$ is carried out similarly to formula (4), in accordance with the $\pi$-theorem [6]. Since the SI contains direct and inverse values, for example, length $\left(L^{1}\right)$ and running length $\left(L^{-1}\right)$, the total number of quantities must be divided by 2 . This is because an object having symmetrical parts can be calculated from its one part, while others are considered informationally empty, and 4 is due to the fact that $L, M, T, \theta$ are used.

Then, the minimum achievable comparative uncertainty $\varepsilon_{L M T \theta}$ is

$$
\varepsilon_{\text {LMTO }}=(\Delta u)_{\text {LMTO }}=846 / 38265+19 / 846=0.0442
$$

Returning to Equation (7) and using (11), we can state the procedure for applying formula (7). Imagine that in the first project, $\gamma_{1}$ dimensionless criteria were used, and in the second, $\gamma_{2}$, and $\gamma_{1}<\gamma_{L M T \theta}<\gamma_{2}$. Then, according to (7), we obtain

$$
\begin{aligned}
\Delta A_{b 1 \gamma} & =\ln \left(\gamma_{\text {LMTO }} /\left(z_{1}^{\prime \prime}-\beta_{1}^{\prime \prime}\right)\right) / \ln 2 \text { (bits) } \\
\Delta A_{b 2 \gamma} & =\ln \left(\gamma_{\text {LMTO }} /\left(z_{2}^{\prime \prime}-\beta_{2}^{\prime \prime}\right)\right) / \ln 2 \text { (bits) }
\end{aligned}
$$

where $\Delta A_{b 1 \gamma}$ and $\Delta A_{b 2 p}$ respectively, is the information content of the first model and the second model compared to the model that takes into account the optimal number of dimensionless criteria.

By analyzing Equations (7) and (13), some readers may suggest that it is pre- 
ferable to use a model with fewer variables when modeling a physical process. However, this is a wrong judgment, and here is why. By comparing $\Delta A_{b 1 \gamma}$ and $\Delta A_{b 2 \gamma}$ in absolute terms, the researcher can "instantly" identify which one is smaller. That is, the number of dimensionless criteria taken into account is closer to the optimal one corresponding to the minimum comparative uncertainty. Thus, a project with a smaller absolute value of $\Delta A_{b}$ is more informative. So, this approach will greatly reduce the time spent by researchers on the analysis of publications.

It can be stated that the above-mentioned formulas may be applied for any TFRS using quantities of SI. It is explained by the fact that the relative and comparative uncertainties of the dimensional quantity $U$ and the dimensionless quantity $u$ are equal:

$$
\begin{aligned}
& \Delta U / S^{*}=(\Delta U / a) /\left(S^{*} / a\right)=(\Delta u / S) \\
& (r / R)=(\Delta U / U) /(\Delta u / u)=(\Delta U / U) \cdot(a / \Delta U) \cdot(U / a)=1
\end{aligned}
$$

where $S$ is the dimensionless interval of changes, and $\Delta u$ is the dimensionless overall absolute uncertainty when measuring the dimensionless quantity $u ; S$ is the dimensional interval of changes, and $\Delta U$ is the dimensional overall absolute uncertainty when measuring the dimensional quantity $U$; $a$ is a scaling factor; $r$ is the relative uncertainty of the dimensional quantity $U$; and $R$ is the relative uncertainty of the dimensionless quantity $u$.

The examples presented below will demonstrate the application of formula (7) and (8) to the analysis of several TFRSs published in recent years.

\section{Factual TFRS and Results}

\subsection{Analysis of Researches}

Articles considered further were not chosen randomly because the objects under study (energy storage systems and ice slurry) have been under the close supervision of the author for more than 42 years. Therefore, the author assumes that he has, at least, the right to express his point of view, even if it does not agree with the opinion of the majority.

\subsubsection{Energy Storage}

Similarity theory was used in formulating a theoretical model and conducting experimental studies on the characteristics of the operation of an accumulation system based on microcapsules with a phase-change material [32]. Experiments were organized on the accumulation of thermal energy for nine variants of surface temperatures. The authors revealed a clear dependence of the amount of stored energy with various dimensionless criteria. The study was conducted within $C o P_{\mathrm{SI}} \equiv L M T \Theta$ using $\gamma^{*}=12$ criteria. Unfortunately, the comparison of theoretical and experimental calculations was not accompanied by any numerical calculations.

Absolute uncertainty was presented for the measured value of temperature $\left( \pm 0.3^{\circ} \mathrm{C}\right)$, and the relative uncertainties were introduced for thermal conductivi- 
ty $( \pm 5 \%)$, specific heat $( \pm 2.22 \%)$, porosity $( \pm 4.03 \%)$, Stefan number $( \pm 7.94 \%)$, supercooling parameter $( \pm 7.21 \%)$, and stored energy through the hot wall $( \pm 8.36 \%)$. The authors declared that "that the deviation between the correlated formula of the dimensionless accumulated energy through hot wall and the measured data is less than $10 \% . "$

It is obvious that, there is no match between $\gamma_{L M T \theta}=19(11)$ and $\gamma^{*}=12$ selected dimensionless complexes.

An additional comment is related to the magnitude of the inaccuracy of the model. Even if we assume that all $\left(\gamma^{*}=12\right)$ criteria are calculated and measured with high accuracy, for example, 2\% (this is very difficult to believe), then the total relative uncertainty $r$ of the key criterion (accumulated energy) will be, at least, equals [18]

$$
r=K \cdot\left(\sum_{i=1}^{N} r_{i}^{2}\right)^{1 / 2}=1.1 \times\left(12 \times 0.02^{2}\right)^{1 / 2}=0.0762
$$

where $N=12, K$ is a correction factor equal to 1.1 for the most important parameters of the devices and monitoring the characteristics of the finished product.

An important point to make here. Unfortunately, many researchers still understand by relative uncertainty the ratio of absolute uncertainty to the value of the measured variable. By its definition, this is true. However, in reality, the achieved relative uncertainty is determined by the measurement limit of the measuring instrument used, in the event that the value of the particular variable under investigation is determined. For another case, when the studied variable is a function of several variables, in the simplest case, the relative uncertainty is calculated by the formula (14). A more detailed analysis of the various formulas used to calculate the relative uncertainty is presented in [18].

This value $(0.0762)$ is very close to the declared value $(0.0836)$. At the same time, due to the lack of calculation of the total absolute experimental uncertainty of the accumulated energy under study, it is impossible to apply the basic formula (8). Thus, in order to improve the reliability of the presented diagrams necessary for designing and calibrating tank sizes and an effective operating mode, researchers are advised to increase the number of parameters taken into account to the recommended one. In addition, if the developers will use more accurate measuring devices and present the integral experimental uncertainty achieved for estimating the accumulated energy with an interval of its changes, it will be possible to calculate the experimental comparative uncertainty and compare it with the theoretically formulated one.

Thus, the stated correlation between theory and experiment may not confirm the plausibility of the presented model, its structure and the stated relationships between the quantities used.

\subsubsection{Heat Process}

Minced fish in the form of a thin layer is frozen on the outer cooled surface of the rotating cylindrical chamber, the axis of which is located horizontally [33]. The model belongs to $C o P_{\mathrm{SI}} \equiv L M T \Theta$. Using the theory of similarity, the au- 
thor presented a theoretical model and the results of experiments in the form of the relationship of dimensionless complexes. The target temperature of the product to be frozen was $\theta_{\mathrm{s}}^{\circ}$, depending on six criteria $\left(z^{\prime \prime}-\beta^{\prime \prime}=6\right)$.

In order to calculate the measurement uncertainty of the main quantity, the author used the following data:

$$
\theta_{\mathrm{s}}^{\circ}=\left(\theta_{\mathrm{s}}-\theta_{\mathrm{e}}\right) /\left(\theta_{\mathrm{cr}}-\theta_{\mathrm{e}}\right)
$$

where $\theta_{\mathrm{cr}}$ is a cryoscopic temperature of a product, $\theta_{\mathrm{cr}}$ varied within $\theta_{\mathrm{crmin}}=-2^{\circ} \mathrm{C}$ to $\theta_{\text {crmax }}=-1^{\circ} \mathrm{C}, \Delta \theta_{\mathrm{cr}}$ is its measurement uncertainty, $\Delta \theta_{\mathrm{cr}}=0.1^{\circ} \mathrm{C}, \theta_{\mathrm{s}}$ is a temperature of outer surface of a product, $\theta_{\mathrm{s}}$ varied within $\theta_{\mathrm{smin}}=-17^{\circ} \mathrm{C}$ to $\theta_{\text {smax }}=-2^{\circ} \mathrm{C}, \Delta \theta_{\mathrm{s}}$ is its measurement uncertainty, $\Delta \theta_{\mathrm{s}}=0.5^{\circ} \mathrm{C}, \theta_{\mathrm{e}}$ is an evaporating temperature of the refrigerant, $\theta_{\mathrm{e}}$ varied within $\theta_{\text {emin }}=-20^{\circ} \mathrm{C}$ to $\theta_{\text {emax }}=-10^{\circ} \mathrm{C}, \Delta \theta_{\mathrm{e}}$ is its measurement uncertainty, $\Delta \theta_{\mathrm{e}}=0.5^{\circ} \mathrm{C}$.

Using recommendations of [34] and (16), we calculated a value of the total experimental uncertainty $\left(\Delta \theta_{\mathrm{s}}^{\circ}\right)_{\exp }$ :

$$
\begin{aligned}
\left(\Delta \theta_{\mathrm{s}}^{\circ}\right)_{\exp }= & \left(\Delta \theta_{\mathrm{s}}+\Delta \theta_{\mathrm{e}}\right) /\left(\left|\theta_{\mathrm{cr}}-\theta_{\mathrm{e}}\right|\right) \\
& +\left|\theta_{\mathrm{s}}-\theta_{\mathrm{e}}\right| /\left(\left(\Delta \theta_{\mathrm{cr}}+\Delta \theta_{\mathrm{e}}\right) \cdot\left|\Delta \theta_{\mathrm{cr}}+\Delta \theta_{\mathrm{e}}\right|^{2}\right) \\
\approx & 0.066
\end{aligned}
$$

From (8), using calculated values $\mu_{\mathrm{SI}}(4)$ and $z^{\prime \prime}-\beta^{\prime \prime}=6, z^{\prime}-\beta^{\prime}=846$ (10), we calculated the theoretical uncertainty $\left(\Delta \theta_{\mathrm{s}}^{\circ}\right)_{\mathrm{pmm}}$ :

$$
\begin{aligned}
\left(\Delta \theta_{\mathrm{s}}^{\circ}\right)_{\mathrm{pmm}} & =\theta_{\mathrm{s}}^{\circ} \cdot\left(\left(z^{\prime}-\beta^{\prime}\right) / \mu_{\mathrm{SI}}+\left(z^{\prime \prime}-\beta^{\prime \prime}\right) /\left(z^{\prime}-\beta^{\prime}\right)\right) \\
& =0.95 \cdot[846 / 38265+6 / 846]=0.028
\end{aligned}
$$

where $\theta_{\mathrm{s}}^{\circ}=\left(\theta_{\text {smax }}-\theta_{\text {emin }}\right) /\left(\theta_{\text {crmax }}-\theta_{\text {emin }}\right)=(-2+20) /(-1+20)=0.95$ is a maximum value of the $\theta_{\mathrm{s}}^{\circ}$ changes interval inherent in the chosen model [33].

Comparing (17) and (18), it can be argued that the experimental uncertainty is much larger than the value calculated by the information method. Therefore, in order to improve the eligibility of the proposed model, it is recommended to increase the accuracy of measuring instruments and the number of complexes in the model of process.

In the proposed example, all successive steps are presented to calculate absolute and comparative uncertainties in order to clarify the feasibility of using the information-oriented approach as a universal tool for assessing the convergence of theoretical calculations and experimental results.

\subsubsection{Ice Slurry Storage}

In [35], heat transfer was studied in a storage tank with ice chips and water in a stationary state. For process modeling, the TRNSYS program was used. The authors compared theoretical and experimental results for the accumulation and disposal of ice in the tank. The model belongs to $C o P_{\mathrm{SI}}=L M T \Theta$. Twenty-one quantities $\left(z_{1}^{\prime \prime}=21\right)$ were taken into account. With, for example, six main quantities $\left(\beta_{1}^{\prime \prime}=6\right)$, the number of dimensionless complexes is $\gamma_{1}=z_{1}^{\prime \prime}-\beta_{1}^{\prime \prime}=15 \quad$ [36]. The authors stated that, based on the results of the validation of the model, it can 
be argued that it satisfactorily predicts the heat and mass exchange processes in the storage tank during the production and disposal of ice. However, the authors did not present any calculations of the achieved uncertainties.

Therefore, it is difficult to agree on the conclusions of the authors. This is because the actual validation process cannot serve as a justification for the proximity and consistency of the theoretical and experimental results [37]. This is why it is not recommended to present the validation results as a tool for assessing the acceptability of the model. To this should be added the fact that the number of chosen complexes of 15 is less than the recommended 19 (11).

In [38], a system of accumulation of liquid ice was investigated, which provided a cooling for a building. The authors carefully analyzed the efficiency of the system, using the concept of exergy and the total number of chosen quantities $z_{2}^{\prime \prime}=35$. However, the authors did not provide a comparison of computer data with experiment. The $C o P_{\text {SI }}$ belong $L M T \Theta$. Assuming that $\beta_{2}^{\prime \prime}=6$, $z_{2}^{\prime \prime}-\beta_{2}^{\prime \prime}=29$.

That is why one can compare two researches [35] [38], with the same CoP, following formula (13)

$$
\begin{aligned}
\Delta A_{b 1 \gamma} & =\ln \left(\gamma_{\text {LMTE }} / \gamma_{1}\right) / \ln 2=0.236 \text { (bits) } \\
\Delta A_{b 2 \gamma} & =\ln \left(\gamma_{\text {LMTO }} / \gamma_{2}\right) / \ln 2=-0.423 \text { (bits) }
\end{aligned}
$$

Comparing the absolute values of $\Delta A_{b 1 \gamma}$ and $\Delta A_{b 2 \gamma},\left|\Delta A_{b 1 \gamma}\right|<\left|\Delta A_{b 2 \gamma}\right|$, it can be

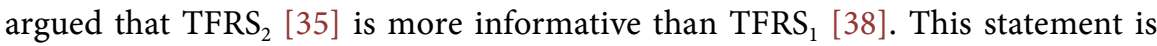
based on the fact that the number of dimensionless criteria in $\mathrm{TFRS}_{2}$ [35] is closer to optimal compared to TFRS $_{1}$ [38]. This approach will facilitate researchers a comparative analysis of scientific and technical publications. In addition, combining it with Equation (8) will allow scientists to increase the credibility of the models being developed and the practical value of the presented results.

At the same time, the final decision on the preference of a particular model cannot be proposed due to the lack of a complete set of experimental data including declaration and calculation of the achieved values of uncertainties. In this situation, the author is forced to note that the recommendations published in two TFRSs would be more impressive if the article presents a comparison of computer data, field test results and general uncertainty achieved for the objective function. However, the introduced analyses show that the current situation is suitable for the design companies projecting refrigeration equipment and spending millions of dollars. Unfortunately, only customers are affected.

\section{Discussion}

This article, to the readers' judgment, presents an information-oriented method and its possible applications to the analysis of the operation of refrigeration systems and installations. Compared with statistical and expert approaches, which are somewhat inherent in the subjectivity and personal philosophical view of the 
researcher, the method presented is theoretically justified. Therefore, in the author's opinion, it can be applied to any models in which quantities from the SI are used.

The proposed formula (8) for calculating the absolute and comparative uncertainties of the formulated model is simple in form and does not require much effort on the part of the developer to apply it. This formula allows you to check the value of the threshold discrepancy [18] between the theoretical model and the experimental data obtained.

The information approach does not contradict the theory of measurement, the achievements of which are obvious in any field of human activity. Its application possible and necessary only at the stage of formulating the model structure. At the subsequent stages, concepts, axioms, principles and formulas of the theory of measurements should be used. The absolute uncertainty calculated by the formula (8) is only an a priori uncertainty before the application of any analytical or numerical methods of calculation, as well as computerization of the model.

Scientists should have a language to indicate that a study or research group provided substantial evidence in favor of a relationship or effect. Comparative uncertainty is a term that serves this purpose. The presented study shows that ignoring comparative uncertainty does not provide much evidence that real relationships were found and scientific statements show a real connection.

This has become something of an independent arms race, in which many organizations seem to remain in the race, at least not to be left behind. Many of the world's technical talents, located in leading institutions, work diligently and have generous resources in modern laboratories to realize their vision of the perfect simulation of refrigeration equipment. Given the above, it is natural to ask the question: when have numerous theoretical studies achieved real results with the lowest cost and proven practical implementation?

The author belongs to a small part of the scientific community, which responds: not in the near future. Having spent many years on developing and commercializing ice generators and energy storage systems, the author developed a very pessimistic view. Without a decisive change in the position of scientists and engineers with regard to a more thorough and balanced calculation of the uncertainties of heat and mass transfer equipment and processes, the gap between the steady increase in the number of published scientific articles and their practical value will increase.

\section{Conclusions}

The information method and its possible applications for the analysis of technical feasibility and study of the operation of refrigeration equipment are presented. This method is theoretically proven and, surprisingly, easy to perceive and explain the results. At its conclusion, no assumptions were made. The method lacks the subjectivity of a researcher who makes decisions in accordance with his knowledge, experience and intuition. The formula (8) for calculating the 
absolute and comparative uncertainties of the model, designed to analyze the operation of refrigeration equipment, is simple in form and does not require much effort on the part of the developer to apply it.

The proximity of theoretical predictions to experimental data is implied by the majority of researchers, as self-evident confirmation of the proposed theoretical concept. However, a "statistical significance" between the results of the approach of an artificial neural network approach or any other type of theoretical model and experimental data is not sufficient evidence of the correctness of the chosen model [39]. A necessary condition for the accuracy of the model is, above all, the smallness of the calculated total uncertainty of the objective function (quantity) compared with the mismatch between the theoretical and experimental values. This, at first glance, the obvious approach has not found due attention in scientific publications devoted to the analysis of energy efficiency of refrigeration units. At the same time, the author hopes to awaken the interest of air-conditioning and refrigeration specialists on this issue.

Comparative uncertainty is a theoretically justified tool that allows scientific groups to provide substantial evidence in favor of the identified relationships or the effect obtained in the course of research. This study suggests that ignoring comparative uncertainty leads to a situation where scientific statements are far from achieving real results, positive or negative.

The achieved results will help engineers and researchers to choose the most suitable models and methods that have comparative uncertainties that are closest to the theoretically recommended ones. This will help increase the likelihood of choosing optimal models of refrigeration equipment.

\section{Conflicts of Interest}

The author declares no conflicts of interest regarding the publication of this paper.

\section{References}

[1] Hensen, J.L.M. (2011) Building Performance Simulation for Sustainable Building Design and Operation. Proceedings of the 60 th Anniversary Environmental Engineering Department, Czech Technical University, Prague, 1-8. http://goo.gl/yYYhLW

[2] Zhu, Y. and Jiang, P. (2014) Experimental and Numerical Investigation of the Effect of Shock Wave Characteristics on the Ejector Performance. International Journal of Refrigeration, 40, 31-42. http://sci-hub.tw/10.1016/j.ijrefrig.2013.11.008 https://doi.org/10.1016/j.ijrefrig.2013.11.008

[3] Wang, S., et al. (2014) Two-Phase Heat Transfer and Pressure Drop of Propane during Saturated Flow Boiling Inside a Horizontal Tube. International Journal of Refrigeration, 41, 200-209. http://sci-hub.tw/10.1016/j.ijrefrig.2013.03.019 https://doi.org/10.1016/j.ijrefrig.2013.03.019

[4] Yu, B., et al. (2018) Performance Improvements Evaluation of an Automobile Air Conditioning System Using $\mathrm{CO}_{2}$-Propane Mixture as a Refrigerant. International Journal of Refrigeration, 88, 172-181. https://doi.org/10.1016/j.ijrefrig.2017.12.016 
[5] Gill, J., Singh, J., Ohunakin, O.S. and Adelekan, D.S. (2018) Artificial Neural Network Approach for Irreversibility Performance Analysis of Domestic Refrigerator by Utilizing LPG with $\mathrm{TiO}_{2}$-Lubricant as Replacement of $\mathrm{R} 134 \mathrm{a}$. International Journal of Refrigeration, 89, 159-176. https://doi.org/10.1016/j.ijrefrig.2018.02.025

[6] Bahman, A.M., Ziviani, D. and Groll, E.A. (2018) Vapor Injected Compression with Economizing in Packaged Air Conditioning Systems for High Temperature Climate. International Journal of Refrigeration, 94, 136-150.

https://doi.org/10.1016/j.ijrefrig.2018.07.024

[7] Lucas, C., Rusche, H., Schroeder, A. and Koehler, J. (2014) Numerical Investigation of a Two-Phase $\mathrm{CO}_{2}$ Ejector. International Journal of Refrigeration, 43, 154-166, http://sci-hub.tw/10.1016/j.ijrefrig.2014.03.003 https://doi.org/10.1016/j.ijrefrig.2014.03.003

[8] Kundu, A., Kumar, R. and Gupta, A. (2014) Comparative Experimental Study on Flow Boiling Heat Transfer Characteristics of Pure and Mixed Refrigerants. International Journal of Refrigeration, 45, 136-147.

http://sci-hub.tw/10.1016/j.ijrefrig.2014.05.023 https://doi.org/10.1016/j.ijrefrig.2014.05.023

[9] Cheung, H. and Braun, J.E. (2016) A General Method for Calculating the Uncertainty of Virtual Sensors for Packaged Air Conditioners. International Journal of Refrigeration, 63, 225-236. http://sci-hub.tw/10.1016/j.ijrefrig.2015.06.022 https://doi.org/10.1016/j.ijrefrig.2015.06.022

[10] Kim, H.S., et al. (2016) Flow Characteristics of Refrigerant and Oil Mixture in an Oil Separator. International Journal of Refrigeration, 70, 206-218. https://doi.org/10.1016/j.ijrefrig.2015.08.003

[11] Tian, Z., Gu, B., Qian, C., Yang, L. and Liu, F. (2015) Electronic Expansion Valve Mass Flow Rate Prediction Based on Dimensionless Correlation and ANN Model. International Journal of Refrigeration, 57, 1-10. https://doi.org/10.1016/j.ijrefrig.2015.04.016

[12] Coquelet, C., Abbadi, J. and Houriez, C. (2016) Prediction of Thermodynamic Properties of Refrigerant Fluids with a New Three-Parameter Cubic Equation of State. International Journal of Refrigeration, 69, 418-436. https://doi.org/10.1016/j.ijrefrig.2016.05.017

[13] Gill, J. and Singh, J. (2017) Adaptive Neuro-Fuzzy Inference System Approach to Predict the Mass Flow Rate of R-134a/LPG Refrigerant for Straight and Helical Coiled Adiabatic Capillary Tubes in the Vapor Compression Refrigeration System. International Journal of Refrigeration, 78, 166-175. https://doi.org/10.1016/j.ijrefrig.2017.02.004

[14] Shah, M.M. (2017) A Correlation for Heat Transfer during Boiling on Bundles of Horizontal Plain and Enhanced Tubes. International Journal of Refrigeration, 78, 47-59. https://doi.org/10.1016/j.ijrefrig.2017.03.010

[15] Wang, S., et al. (2018) Vapor-Liquid Equilibrium and Molecular Simulation Data for Carbon Dioxide $\left(\mathrm{CO}_{2}\right)+$ Trans-1,3,3,3-Tetrafluoroprop-1-Ene (R-1234ze(E)) Mixture at Temperatures from 283.32 to $353.02 \mathrm{~K}$ and Pressures up to 7.6 MPa. International Journal of Refrigeration, 98, 362-371. ttps://doi.org/10.1016/j.ijrefrig.2018.10.032

[16] Ho, C.J., Chen, C.C. and Yan, W.M. (2016) Experimental and Numerical Study on Transient Thermal Energy Storage of Microencapsulated Phase Change Material Particles in an Enclosure. International Journal of Heat and Mass Transfer, 94, 191198. http://sci-hub.tw/10.1016/j.ijheatmasstransfer.2015.11.055 
https://doi.org/10.1016/j.ijheatmasstransfer.2015.11.055

[17] ASME V\&V 10-2006 (2006) Guide for Verification and Validation in Computational Solid Mechanics. The American Society of Mechanical Engineers Standards 2006, 1-53.

[18] Rabinovich, S. (2017) Guide to the Expression of Uncertainty in Measurement. 3rd Edition, Springer International Publishing, New York, 85. https://goo.gl/5bofbE

[19] Christie, M.A., et al. (2005) Error Analysis and Simulations of Complex Phenomena. Los Alamos Science, 29, 1-20. http://www2.stat.duke.edu/ fei/samsi/Readings/DHigdon/lascience.pdf

[20] Deepak, B. and Wright, M. (2006) Uncertainty Analysis of Laminar Aero-Heating Predictions for Mars Entries. Journal of Thermophysics and Heat Transfer, 20, 652-662. https://doi.org/10.2514/1.20993

[21] Menin, B. (2019) A Look at the Uncertainty of Measuring the Fundamental Constants and the Maxwell Demon from the Perspective of the Information Approach. Global Journal of Researchers in Engineering: A Mechanical and Mechanics Engineering, 19, 1-17.

https://globaljournals.org/GJRE_Volume19/1-A-Look-at-the-Uncertainty.pdf https://doi.org/10.34257/GJREAVOL19IS1PG1

[22] Menin, B. (2018) $h, k$, NA: Evaluating the Relative Uncertainty of Measurement. American Journal of Computational and Applied Mathematics, 8, 93-102. http://article.sapub.org/10.5923.j.ajcam.20180805.02.html

[23] Menin, B. (2018) Bekenstein-Bound and Information-Based Approach. Journal of Applied Mathematics and Physics, 6, 1675-1685. http://file.scirp.org/pdf/JAMP_2018082114292484.pdf https://doi.org/10.4236/jamp.2018.68143

[24] Menin, B. (2017) Information Measure Approach for Calculating Model Uncertainty of Physical Phenomena. American Journal of Computational and Applied Mathematics, 7, 11-24. https://goo.gl/m3ukQi https://doi.org/10.3390/IS4SI-2017-04034

[25] Sonin, A.A. (2001) The Physical Basis of Dimensional Analysis. 2nd Edition, MIT, Cambridge, MA. http://goo.gl/2BaQM6

[26] Sedov, L.I. (1993) Similarity and Dimensional Methods in Mechanics. 10th Edition, CRC Press, London.

[27] Bich, W. (2019) The Third-Millennium International System of Units. Rivista del Nuovo Cimento, 42, 49-102. http://www.roma1.infn.it/ luci/LabSS/new_SI_system.pdf

[28] NIST Special Publication 330 (SP330) The International System of Units (SI) 2008. https://nvlpubs.nist.gov/nistpubs/SpecialPublications/NIST.SP.330-2019.pdf

[29] Menin, B. (2015) Possible Limits of Accuracy in Measurement of Fundamental Physical Constants. International Referred Journal of Engineering and Science, 4, 8-14. http://goo.gl/HjYBOs

[30] Brillouin, L. (2004) Science and Information Theory. Dover, New York.

[31] Volkenstein, M.V. (2009) Entropy and Information. Birkhauser Verlag AG, Basel, Berlin. https://goo.gl/eHhRvd

[32] Ho, C.J., Chen, C.C. and Yan, W.M. (2016) Experimental and Numerical Study on Transient Thermal Energy Storage of Microencapsulated Phase Change Material Particles in an Enclosure. International Journal of Heat and Mass Transfer, 94, 191-198. http://sci-hub.tw/10.1016/j.ijheatmasstransfer.2015.11.055 
https://doi.org/10.1016/j.ijheatmasstransfer.2015.11.055

[33] Menin, B. (2014) Comparative Error of the Phenomena Model. International Referred Journal of Engineering and Science, 3, 68-76. http://goo.gl/DwgYXY

[34] Taylor, J. (1982) An Introduction to Error Analysis. Chapter 1, University Science Books, Mill Valley. https://doi.org/10.1007/978-3-642-19565-5

[35] Tamasauskas, M., Poirier, R., Zmeureanu, R. and Sunyé, R. (2012) Development of a Mathematical Model for the Simulation of a Non-Agitated Ice Slurry Storage Tank. Proceedings of eSim 2012: The Canadian Conference on Building Simulation, 380-392. https://goo.gl/vgi1M2

[36] Yarin, L. (2012) The Pi-Theorem, Experimental Fluid Mechanics. Springer-Verlag, Berlin. https://goo.gl/dtNq3D

[37] Oreskes, N., Shrader-Frechette, K. and Belitz, K. (1994) Verification, Validation and Confirmation of Numerical Models in the Earth Sciences. Science, 263, 641-646. https://doi.org/10.1126/science.263.5147.641

[38] Acar, C. and Dincer, I. (2016) Energy and Exergy Analyses of a Residential Cold Thermal Energy Storage System. International Journal of Exergy, 19, 441-458. https://doi.org/10.1504/IJEX.2016.075879

[39] Wasserstein, R.L., Allen, L. and Lazar, A. (2019) Moving to a World beyond " $p<$ 0.05". The American Statistician, 73, 1-19.

https://www.tandfonline.com/doi/pdf/10.1080/00031305.2019.1583913?needAccess $=$ true

https://doi.org/10.1080/00031305.2019.1583913 